\title{
Radiative transfer in the dusty, irradiated expanding atmospheres of close binary components
}

\author{
M. Srinivasa Rao $^{1}$ and A. Peraiah ${ }^{2}$ \\ 1 Indian Institute of Astrophysics, Bangalore-560034, India \\ e-mail: msrao@iiap.ernet.in \\ $257,4^{\text {th }}$ Cross, $36^{\text {th }}$ Main, BTM $1^{\text {st }}$ Stage, Madiwala-Dollar Scheme, Bangalore 560068, India
}

Received April 25; accepted June 13, 2000

\begin{abstract}
We studied the formation of lines in the irradiated expanding dusty atmospheres of the components of close binary systems. We considered a two-level atom approximation in Non-LTE situation with complete redistribution. The thickness of the atmosphere is set to be equal to twice that of the stellar radius. Maximum expansion velocities is set to be as large as 50 mean thermal units. The isotropically scattering dust is distributed uniformly in the atmosphere. We have computed the lines using a total optical depths $10^{4}$ at the line centre. The irradiation from the secondary is one, five and ten times the self radiation. The line fluxes in the line of sight are calculated by using the total source function which is the sum of the source functions due to self radiation and that due to irradiation from the secondary. The line profiles formed in dusty atmosphere are compared with those formed in dust free atmosphere. The profiles are presented for different velocities of expansion, different separations between the components and several dust optical depths.

$\mathrm{P}$ Cygni type profiles are produced in the expanding atmosphere and the irradiation from the secondary enhances the emission in the lines. The major effect of dust on the formation of lines in the expanding atmospheres of the component of close binary is to scatter photons into the line core, among others.
\end{abstract}

Key words: radiative transfer - close binaries reflection effect - dust

\section{Introduction}

In close binary systems light curves, radial velocity curves and line profiles are the most important sources of information to find the physical parameters of the components.

Send offprint requests to: M. Srinivasa Rao
The atmosphere of the component of a close binary system are distorted by the tidal effect due to the presence of its companion, and self rotation. Nonuniform temperature distribution over its surface (which may arise due to gravity darkening), and the additional heating of the stellar surface by incident radiation from the companion star (particularly important for X-ray binaries), eclipses, stellar wind, and interaction effects associated with circumstellar gas structures (disks, jets, shells etc.) will change the radiation field in the atmosphere.

Theoretical studies about the reflection effect using actual model atmospheres are more recent. All the three possible combinations (i.e., like when primary and the secondary components are hot and hot, cool and cool, and hot and cool) are studied by Buerger (1969, 1972), Nordlund \& Vaz (1990), Claret \& Gemenz (1992) respectively. Vaz (1985) \& Wilson (1990) reviewed several aspects of reflection effect. They found that irradiation from the secondary component will effect the lines and as well as equivalent widths. They also found that theoretical bolometric albedos have been found to be in good agreement with observations. Peraiah \& Srinivasa Rao (1983) studied effects of reflection on formation of spectral lines in a purely scattering atmosphere and how the equivalent widths change due to irradiation from the secondary. However these calculations were done in static atmospheres. Recently Peraiah \& Srinivasa Rao (1998, hereafter referred to as Paper I) have studied this problem of irradiation on line formation in an expanding atmosphere of the component of a close binary system and P-Cygni type profiles are found to be produced. The study of line formation in the dusty envelopes remains practically unexplored. The presence of dust is revealed in the infrared observations of many stellar objects, like gaseous nebulae, active galactic nuclei, T-Tauri stars and binary stars. Presence of dust, radial expansion, geometrical extension, chemical composition etc. are some of the physical and geometrical properties one should include in any serious calculation 
of line formation in such atmospheres. The purpose of this study is to compute the effects of dust on the formation of lines in the expanding atmospheres with light of the secondary falling on the component in a close binary system. We consider that the dust scatters radiation isotropically and neither dust absorption nor emission are taken into account.

\section{The equation of transfer in comoving frame in a dusty atmosphere}

The equation of line transfer in the comoving frame with absorption and emission due to dust and gas (see Peraiah \& Wehrse 1978; Peraiah 1984; Wehrse \& Kalkofen 1985) is given by,

$$
\begin{aligned}
& \mu \frac{\partial I(x, \mu, r)}{\partial r}+\frac{\left(1-\mu^{2}\right)}{r} \frac{\partial I(x, \mu, r)}{\partial \mu}=K(x, r) S_{\mathrm{L}}(r) \\
+ & K_{\mathrm{c}}(r) S_{\mathrm{c}}(r)-\left[K(x, r)+K_{\mathrm{c}}(r)\right] I(x, \mu, r) \\
+ & {\left[\left(1-\mu^{2}\right) \frac{V(r)}{r}+\mu^{2} \frac{\mathrm{d} V(r)}{\mathrm{d} r}\right] \frac{\partial I(x, \mu, r)}{\partial x} } \\
+ & K_{\text {dust }} S_{\text {dust }}(r, \mu, x)-I(r, \mu, x),
\end{aligned}
$$

and

$$
\begin{aligned}
& -\mu \frac{\partial I(x,-\mu, r)}{\partial r}-\frac{\left(1-\mu^{2}\right)}{r} \frac{\partial I(x,-\mu, r)}{\partial \mu}=K(x, r) S_{\mathrm{L}}(r) \\
& +K_{\mathrm{c}}(r) S_{\mathrm{c}}(r)-\left[K(x, r)+K_{\mathrm{c}}(r)\right] I(x,-\mu, r) \\
& +\left[\left(1-\mu^{2}\right) \frac{V(r)}{r}+\mu^{2} \frac{\mathrm{d} V(r)}{\mathrm{d} r}\right] \frac{\partial I(x,-\mu, r)}{\partial x} \\
& +K_{\text {dust }} S_{\text {dust }}(r, \mu, x)-I(r, \mu, x),
\end{aligned}
$$

where all the symbols have their respective usual meanings (See Paper I). Further, $K_{\text {dust }}(r)$ is the absorption coefficient of the dust and the dust source function $S_{\text {dust }}(r, \pm \mu, x)$ is given by,

$$
\begin{aligned}
S_{\text {dust }}(r, \pm \mu, x) & =(1-\omega) B_{\text {dust }} \\
& +\frac{\omega}{2} \int_{-\infty}^{+\infty} P\left(\mu, \mu^{\prime}, r\right) I\left(r, \mu^{\prime}, x\right) \mathrm{d} \mu^{\prime}
\end{aligned}
$$

where $B_{\text {dust }}$ is the Planck function for the dust emission, $\omega$ the albedo of the dust and $P$ the isotropic and coherent scattering phase function. The quantity $B_{\text {dust }}$ is normally neglected because the re-emission will be far away from the line centre and therefore may not contribute to the line radiation. Although we need not consider the term containing $B_{\text {dust }}$, we have included it for the sake of completeness.

We have adopted the "CELL" method described by Peraiah (1984) to solve the Eqs. (1)and (2); This is done by suitable discretization in frequency, angle as well as radius. The details of the method was given in Peraiah et al. (1987).

\section{Boundary conditions}

The atmosphere in question is divided into $n$ shells where $n=1$ corresponds $\tau=T$ and $n=100$ corresponding to $\tau=0$, and $\tau$ is the optical depth at any point and $T$ is the total optical depth. The total optical depth is set in advance. We assume that no radiation is incident from outside the spherical shell at $r=B$ and $\tau=0$, while radiation of unit intensity is incident at $r=A, \tau=\tau_{\max }=T$, in the case of purely scattering medium. Thus we have,

$$
\begin{aligned}
& \mathbf{U}^{-}\left(\tau=T, \mu_{j}\right)=1 \\
& \mathbf{U}^{+}\left(\tau=0, \mu_{j}\right)=0, \quad(\epsilon=0, \beta=0)
\end{aligned}
$$

and in the case of the boundary condition of the frequency derivative, we have:

$\frac{\partial \mathbf{U}}{\partial X} \quad\left(\right.$ at $\left.X=\left|X_{\max }\right|\right)=0$.

We have set the velocities at $r=A$ as $V_{A}$, and at $r=B$ as $V_{B}$ which is set equal to 0 and $50 \mathrm{mtu}$. If the spherical shell expands with constant velocity, we have;

$V_{A}=V_{B}$

if there are velocity gradients, then we have;

$V_{A}=0 \quad V_{B}>0$.

Finally we calculate total source function $S$ by adding the source function (1) due to reflected radiation $S_{\mathrm{d}}$ and due to the self radiation $S_{\mathrm{s}}$ (See Paper I)

$S=S_{\mathrm{s}}+S_{\mathrm{d}}$.

We calculate the set of source functions at the points of intersection of the ray parallel to the line of sight and the shell boundaries. These source functions are used to calculate the emergent specific intensities at infinity (or at the observer's point), by using the formula (see Peraiah \& Srinivasa Rao 1983)

$I_{n+1}(r)=I_{0}(n) \mathrm{e}^{-\tau}+\int_{o}^{\tau} S(t) \mathrm{e}^{-[-(\tau-t)]} \mathrm{d} t$,

where $I_{n}(r)$ corresponds to the specific intensity of the ray passing through the shell bounded by $r_{n}$ and $r_{n+1}$ corresponding to perpendicular to the axis $\mathrm{OO}^{\prime}$ (See Fig. 1 of Paper I) at different radii. $I_{0}(n)$ corresponds to the incident intensity at the boundary of the shell and $\tau$ is the optical depth in the sector along the ray path. The source function $S(t)$ is calculated by linear interpolation between $S\left(t_{n}\right)$ and $S\left(t_{n+1}\right)$. The specific intensity at the boundary of each shell is calculated by using Eq. (9).

The incident radiation at $Q$, the bottom of the atmosphere, (See Fig. 1 of Paper I) is given as

$I_{\mathrm{s}}\left(\tau=T, \mu_{j}\right)=1$.

The incident radiation from the secondary is given in terms of $I_{\mathrm{s}}$ in the ratio $I$, where $I$ is given by

$I=\frac{U_{1}}{I_{\mathrm{s}}}$. 

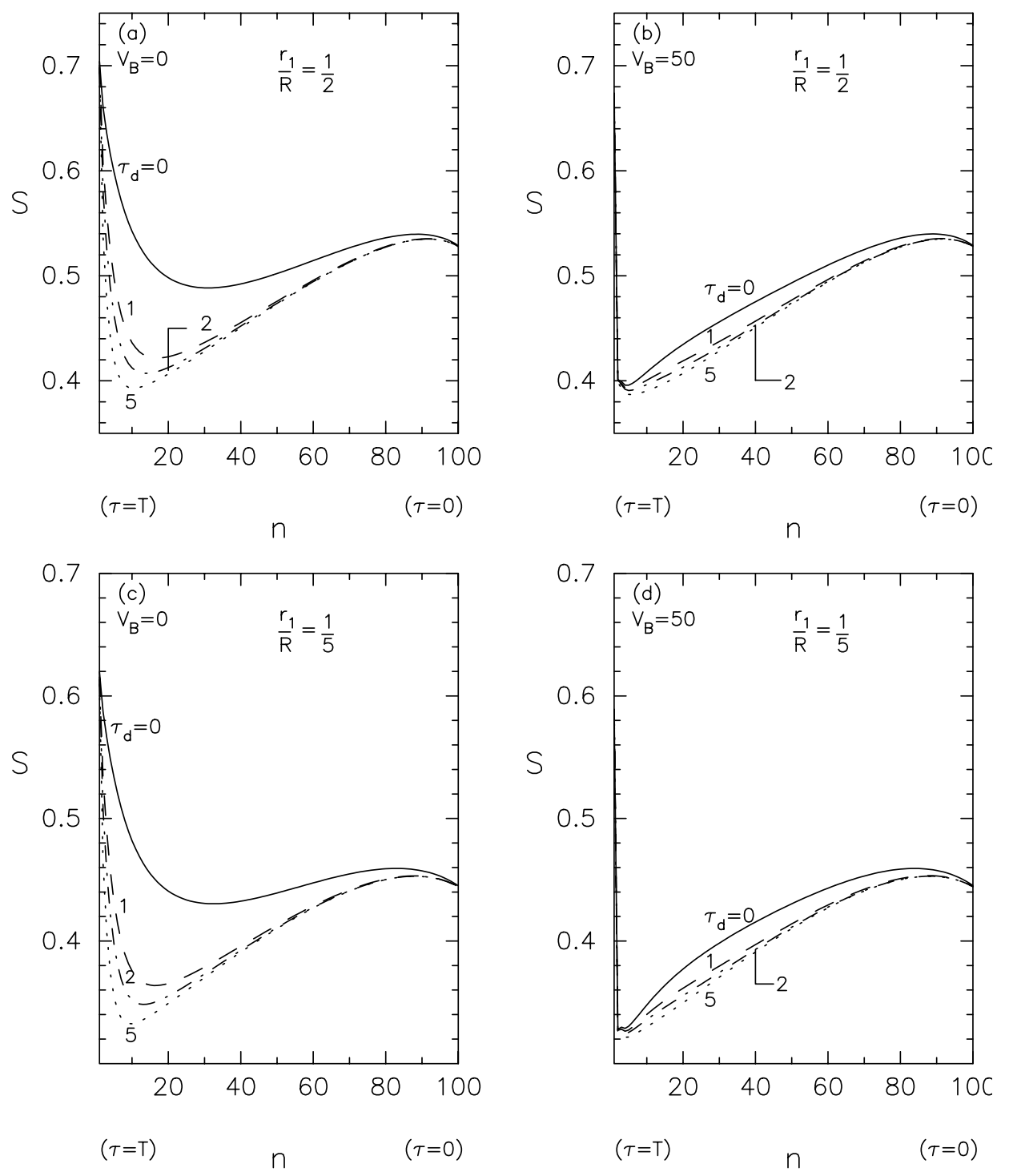

Fig. 1. The source functions $S$ are shown with respect to the shell numbers in a scattering medium for different dust optical depths, velocities, with $\frac{r_{1}}{R}=\frac{1}{2}, \frac{1}{5}$ and $I=5$

The velocities of expansion of the gas are expressed in terms of mean thermal units $V_{\mathrm{T}}(\mathrm{mtu})$ given by,

$V_{\mathrm{T}}=\left[\frac{2 k T_{1}}{m_{i}}\right]^{\frac{1}{2}}$

where $k$ is the Boltzmann constant and $T_{1}$ is the temperature and $m_{i}$ is the mass of the ion. The velocity at $n=1$ or $\tau=T$ is $V_{A}$ and $V_{B}$ is the velocity at $n=100$ or $\tau=0$ are given in terms of mtu or $V_{\mathrm{T}}$ as

$V_{A}=\frac{v_{A}}{V_{\mathrm{T}}}$

$V_{B}=\frac{v_{B}}{V_{\mathrm{T}}}$

where $v_{A}$ and $v_{B}$ are the velocities at the inner radius $A$, outer radius $B$ at radial point $r$ respectively in units of mtu of the gas. We assume uniform expansion of the gases for the sake of simplicity. The proximity of the component is measured in terms of separation parameter $\frac{r_{1}}{R}$, where $r_{1}$ is the radius of the primary and $R$ is the separation of centre of gravity of the two components. The ratio of the outer to the inner radii $\frac{B}{A}$ of the atmosphere is always taken to be 2 . The actual thickness in the components could be much larger than what we have considered here. As the number of parameters is large, we restricted our calculations to this modest thickness of the atmosphere. The line profile fluxes $\frac{F_{Q}}{F_{c}}$ are plotted against the normalized frequency $Q$, where

$Q=\frac{x}{x_{\max }}$,

$F_{Q}=F\left(x_{Q}\right)$,

$F_{C}=F\left(x_{\max }\right)$, 
and

$x=\left(\nu-\nu_{0}\right) / \Delta \nu_{\mathrm{D}}$,

$x_{\max }=|x|+V_{B}$,

$\Delta \nu_{\mathrm{D}}=\nu_{0} \frac{v_{\mathrm{T}}}{C}$,

$x$ lies between \pm 5 units. The equivalent widths are calculated by the relation,

$E Q . W=\int_{x_{\min }}^{x_{\max }}\left(1-\frac{F_{Q}}{F_{c}}\right) \mathrm{d} x$

where

$x_{\min }=-\left(|x|+V_{B}\right)$.

\section{Parameters for computations}

The equations of line transfer given in the Eqs. (1) and (2) are solved following the procedure described in Peraiah et al. (1987). The optical depths along these segments $P \tau$ $P \tau^{\prime}$ are calculated using the Eq. (1) of Paper I. We set $\sigma$ as the electron scattering coefficient equal to (Thomson cross section) to $6.652510^{-25} \mathrm{~cm}^{2}$. The lengths of the segments change between 0 and $2 r$ where $r$ is the radius of the component. We have set an electron density of $10^{14} \mathrm{~cm}^{-3}$. The maximum optical depth is 97.5 while optical depth of the segment along the $x$-axis $\mathrm{OO}^{\prime}$ is 66.525 where the radius of the star is taken to be $10^{12} \mathrm{~cm}$ and the thickness of the atmosphere as $10^{12} \mathrm{~cm}$. The parameters that are used in the calculations are listed below.

$\frac{B}{A}=$ ratio of the outer to the inner radii of the atmosphere of the primary component and whose reflection effect is being studied $(=2)$;

$n=$ number of shells into which the atmosphere of the component is divided;

$\frac{r_{1}}{R}=$ ratio of the radius of the component to that of the line joining the centre of gravity of the two components $r_{1}=210^{12} \mathrm{~cm}$;

$V_{A}=$ initial velocity of expansion in units of mtu at $n=1$ (see Eqs. (12) and (13));

$V_{B}=$ final velocity in units of mtu at $n=100$ (see

Eq. (14));

$S=$ total source function (see Eq. (8));

$S_{\mathrm{s}}=$ source function due to self radiation;

$I=$ ratio of incident radiation to that of self radiation of

the star (see Eq. (11));

$\epsilon=$ probability per scatter that a photon is thermalised

by collisional de-excitation;

$\beta=$ ratio of absorption coefficient in continuum to that in the line;

$T=$ total optical depth;

$Q=\frac{x}{x_{\max }}$ (see Eq. (15));

$\frac{F_{Q}}{F_{\mathrm{c}}}=$ ratio of the line flux at the normalized frequency

$Q^{c}$ to that in the continuum or at $x_{\max }$ (see Eqs. (16) and $(17))$,

$(R ; N . R)=$ with reflection; with no reflection;
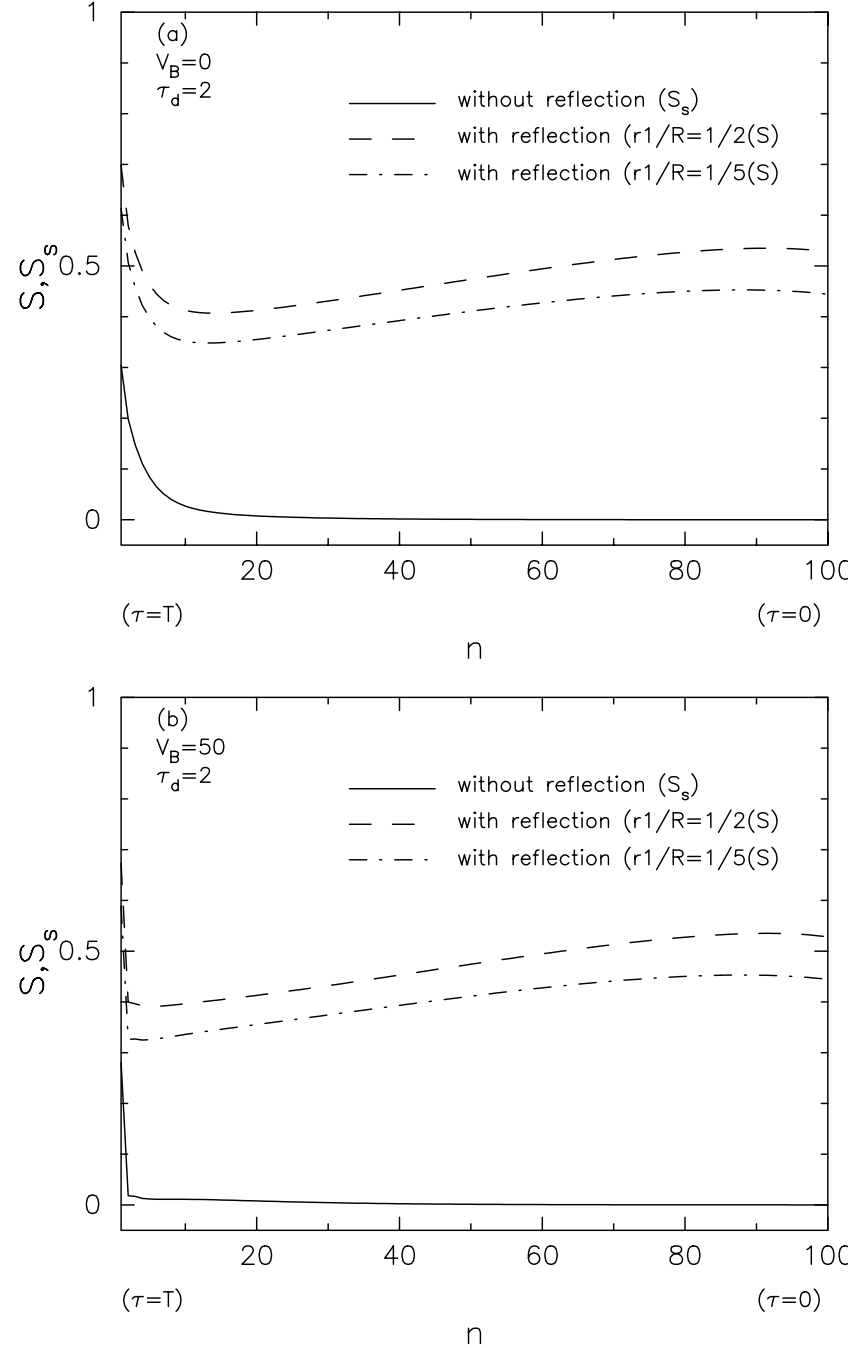

Fig. 2. A comparison of the source function $S$ and $S_{\mathrm{s}}$ are shown with respect to the shell numbers in a scattering medium for a static medium and for a expanding medium when the dust optical depth $\tau_{\mathrm{d}}=2$ and $\frac{r_{1}}{R}=\frac{1}{2}$ and $\frac{1}{5}$

$\frac{H_{\mathrm{e}}}{H_{\mathrm{a}}}=$ height of the emission to the depth of absorption in the line;

$T_{1}=$ temperature in the atmosphere;

$\tau_{\mathrm{d}}=$ optical depth due to dust scattering (isotropic).

Our objective is to compute the effects of the presence of dust on the formation of the spectral lines. The number of parameters is large and this results in a large amount of output consequently we choose few sample parameters to show the effects of dust on the formation of lines. We have chosen trapezoidal points for frequency integration. We employed nine frequency points $(I=9)$ with one frequency point at the centre of the line and two angles $(m=2)$ in each half space which gives a matrix size of $(18 \times 18)$. With this size of frequency angle mesh, we obtain a step size which gives solution to the machine accuracy. The condition of flux conservation in a conservatively scattering medium $(\epsilon=0, \beta=0, \omega=1)$, is maintained. 

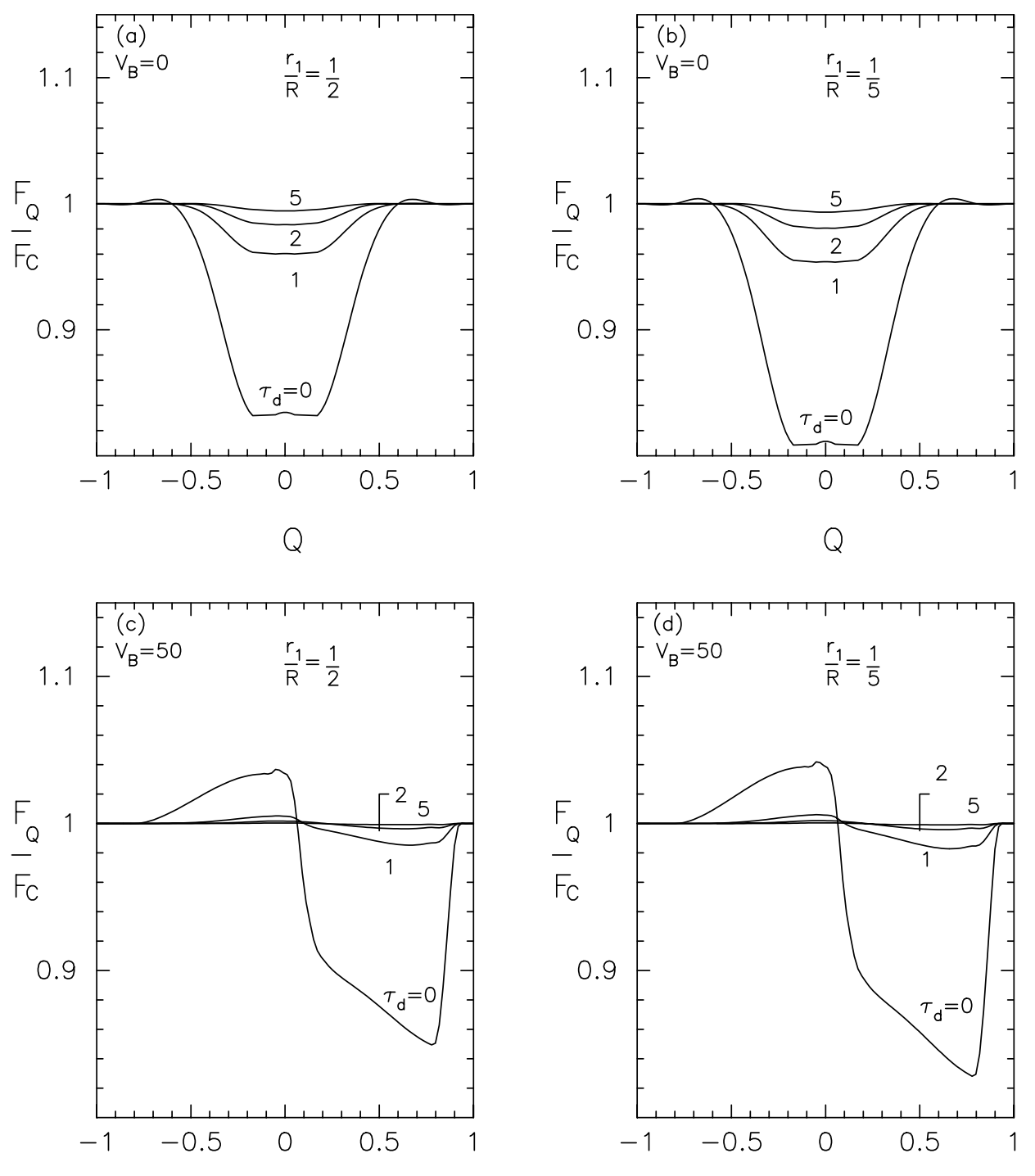

Q

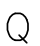

Fig. 3. Comparison of line profiles for different dust optical depths when $\frac{r_{1}}{R}=\frac{1}{2}$ and $\frac{1}{5}$ and for $V_{B}=50$

\section{Result and discussion}

Results are presented in Figs. 1 to 7 for different parameters. The figures are self explanatory regarding the parameters used. The atmosphere of the primary is divided into 100 shells. The separation of the components $\frac{r_{1}}{R}$ where $r_{1}$ is the radius of the primary component and $R$ is the separation of the centers of gravity of the components. We have considered two cases of separation $\frac{r_{1}}{R}=\frac{1}{2}$ and $\frac{1}{5}$, and the atmosphere extension $\frac{B}{A}=2$. The total radial optical depth $T$ is taken to be $10^{4}$. The velocities of expansion are measured in terms of mean thermal units and uniform expansion velocity law is assumed. If $V_{A}$ and $V_{B}$ are the velocities at $A$ and $B$ respectively, then the velocity at any shell boundary $V_{n}=V_{A}+\left[\frac{V_{B}-V_{A}}{N} \times n\right]$. At $A\left(\tau=\tau_{\max }=T\right)$ the velocity is $V_{A}$ and at $B(\tau=0)$ the velocity is $V_{B}$. The parameter $\epsilon<1$ for non-LTE line formation and this is set to equal to 0 . The quantity $\beta$ is set equal to 0 in our calculations. The dust optical depth is taken be $\tau_{\mathrm{d}}=0,1,2,5$. The expansion velocities are taken $V_{B}=0,5,25,50$. But the results are presented for static medium with $V_{A}=0, V_{B}=0$ and expanding medium with $V_{A}=0, V_{B}=50$. The irradiation from the secondary component is taken to be $I=1,5,10$ times the self radiation of the star. However the results are presented for $I=5$ only.

In Fig. 1a, the source function $S$ are plotted for a static dusty medium, with $\frac{r_{1}}{R}=\frac{1}{2}$ and $I=5$ when $\tau_{\mathrm{d}}=0$ (dust free medium), the source function falls rapidly in magnitude from the boundary $\tau=T$ towards the boundary $\tau=0$. It reaches a minimum at around $n=20$ at which 

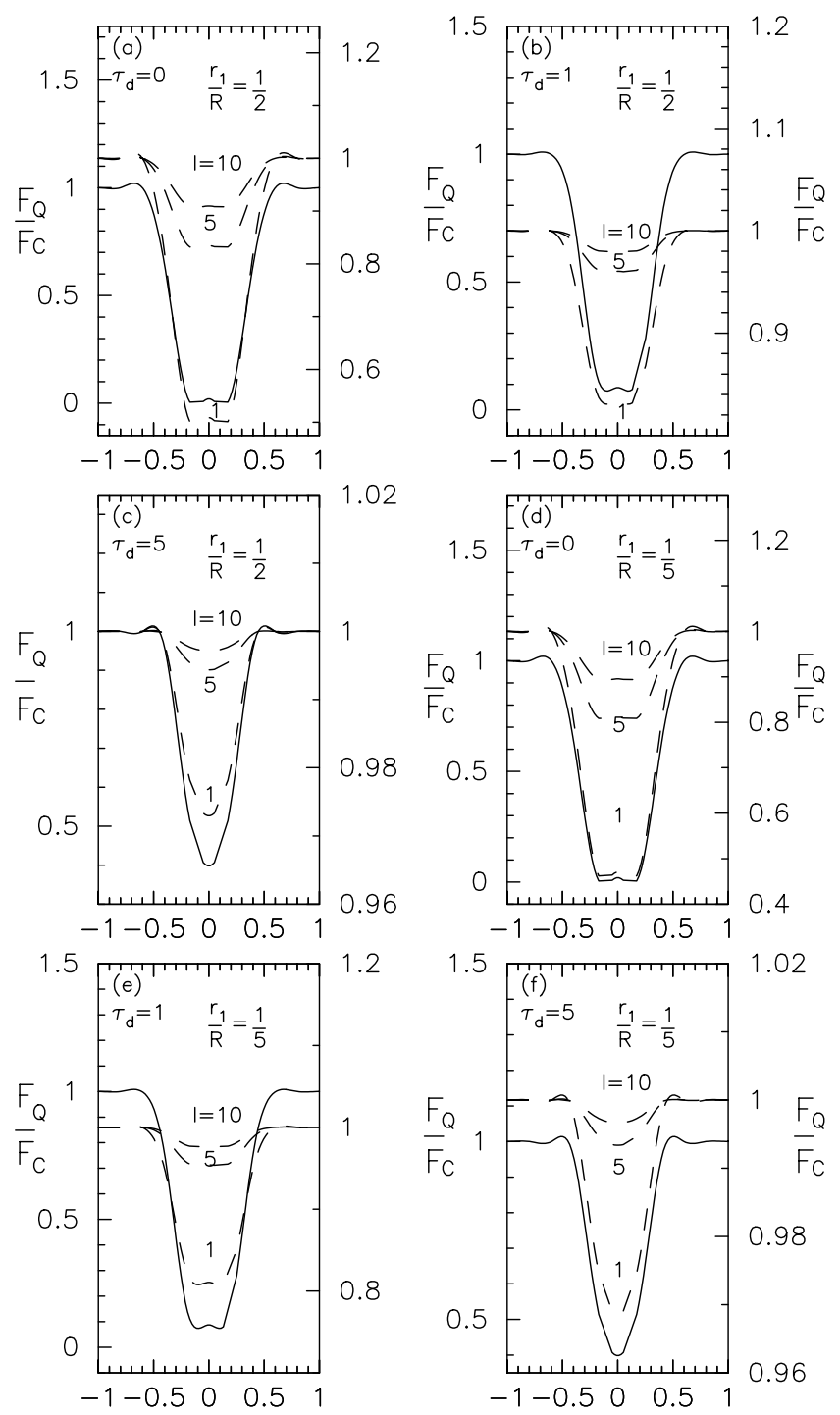

Q

Q

Fig. 4. Comparison of line profiles for different irradiation $(I=$ $1,5,10)$ when $\frac{r_{1}}{R}=\frac{1}{2}$ and $\frac{1}{5}$ for different dust optical depths

point it starts to raise because of the diffuse radiation incident from the secondary. It reaches a maximum at about $n=80$. This is due to the diffuse reflection of the incident light from the secondary by the gaseous medium immediately inside the atmosphere at the boundary at $\tau=0$. When dust is introduced the source functions have been reduced proportionately to the amount of dust present in it but nature of variation of the source function remain the same. The reduction in the source functions in dusty medium is due to the physical effect that dust removes photons from the radiation field and that these are not replaced by emission. In Fig. 1b we introduced expansion with $V_{B}=50 \mathrm{mtu}$. The source functions fall more rapidly near the boundary $\tau=T(n=1)$ but otherwise the variation is similar to those shown in the case of static medium (Fig. 1a). Figures 1c and 1d are the same as those
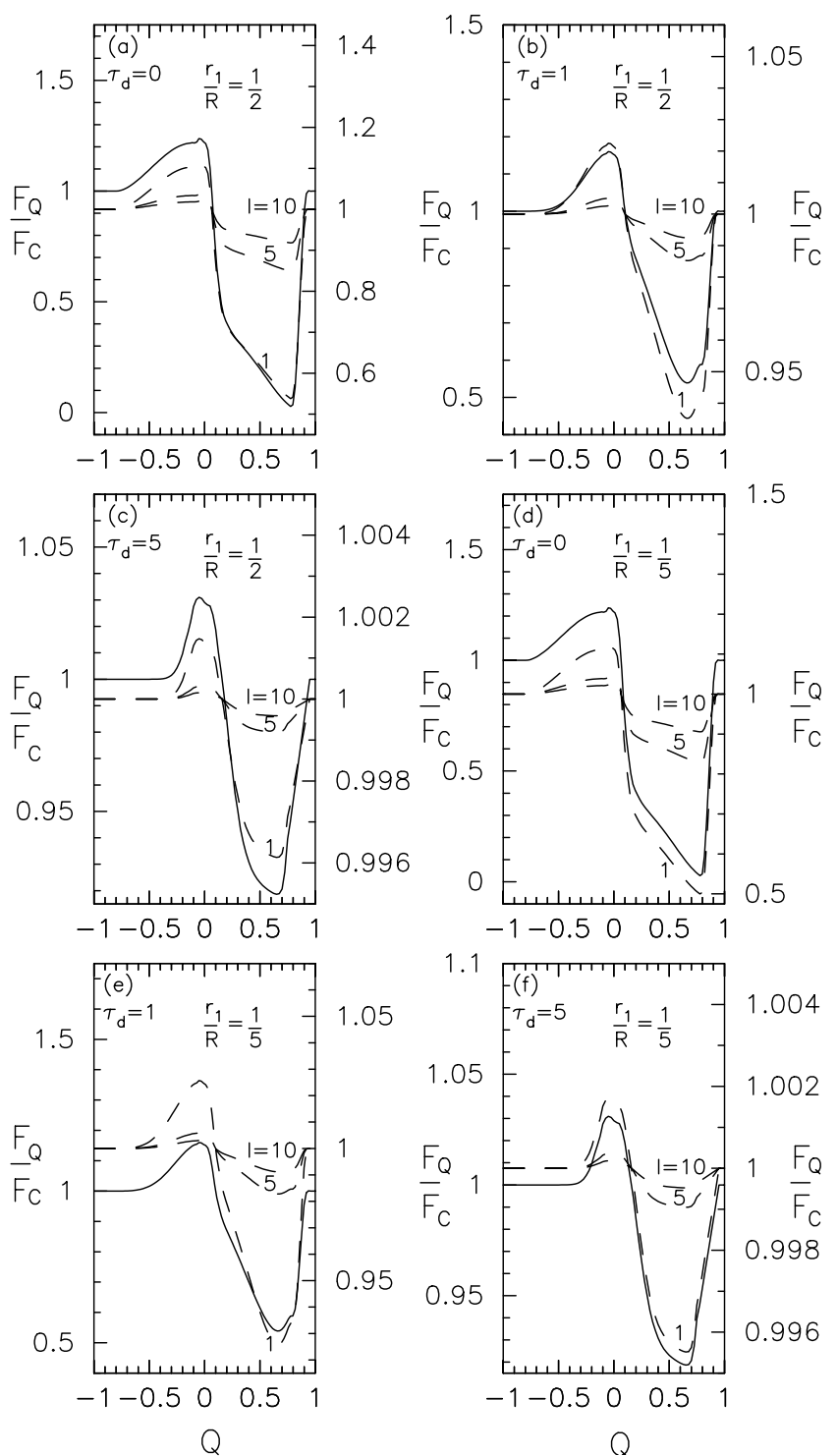

Fig. 5. Same as Fig. 4 but $V_{B}=50$

given in Figs. 1a and $1 \mathrm{~b}$ respectively but with $\frac{r_{1}}{R}=\frac{1}{2}$ replaced by $\frac{1}{5}$. In both the situations there is no change in variation of the source functions but the values of the source functions are much smaller because of the fact that the distance between the components is increased and therefore the incident radiation is reduced by an approximate factor of $\frac{1}{\left(R-r_{1}\right)^{2}}$.

Figures 2a,b gives the source function $S_{\mathrm{s}}$ and $S$ given in Eq. (8) for various parameters shown in the figure, across the atmosphere for $n=1$ to $n=100$. Figure 2a represents a static and scattering medium with $V_{A}=V_{B}=0$ and $\epsilon=\beta=0$ and the incidence radiation factor $I=5$ (see Eq. (10)). The source function $S_{\mathrm{s}}$ decreases slowly from the point $\tau=T$ to the point $\tau=0$ in the scattering medium, with $T=10^{4}$. When the reflected radiation is included, the source function for $\frac{r_{1}}{R}=\frac{1}{2}$ and $\frac{1}{5}$ are considerably enhanced as these source functions 
include the incident radiation from the companion. Similar trend is observed in Fig. 2b when the expansion velocity is $V_{B}=50$.

In Figs. 3a-d the line fluxes are plotted against the normalized frequency points $Q$ (see Eq. (15)) along the line of sight. Each one of the graphs in Figs. 3a-d gives the line profiles for different $\tau_{\mathrm{d}}$ 's. Figures $3 \mathrm{a}$ and $3 \mathrm{~b}$ represent the static atmosphere but with $\frac{r_{1}}{R}=\frac{1}{2}$ and $\frac{1}{5}$ respectively. As the fluxes are normalized, there do not seem to be any change in the symmetric profiles. However, the effect of dust is significant in that dust scatters more photons into line core thereby increasing the emission in the core of the line, and more emission in the cores of the lines formed in the former case (i.e.) $\frac{r_{1}}{R}=\frac{1}{2}$ appears and this is because more light falls on the component in the former case than in the latter case. Figures $3 \mathrm{c}$ and $3 \mathrm{~d}$ give the flux profiles for the same parameters as those in Figs. 3a and 3b respectively except that a velocity of expansion $V_{B}=50 \mathrm{mtu}$ is introduced into the medium. The profiles show asymmetry with red emission and blue absorption and a blue shift of the centre of the line - a P Cygni type profile. The part played by the dust is similar to that shown in Figs. 3a and $3 \mathrm{~b}$. It scatters more photons into the line centre and at high dust optical depth, the line may even disappear altogether.

In Figs. 4a-f line profiles without reflection are compared with those formed with the incident radiation from the secondary component i.e., $I=1,5,10$. The line profiles are plotted with normalized frequency points $Q$, and the ratios of the flux $\frac{F_{Q}}{F_{C}}$. The solid curves (scale given on the right side of the figure) are for the case without irradiation while the dashed are the ones formed with irradiation from the secondary. As the medium is static, the profiles are symmetric with central absorption. Figures $4 \mathrm{a}-\mathrm{f}$ give a comparison of profiles formed in the condition of no irradiation and those with irradiation from the secondary component, and each of the six figures drawn for different $\tau_{\mathrm{d}}$ 's are shown in the respective figures. Each figure contains profiles for $I=0,1,5$ and 10 where 0 means no incident radiation. It appears that the higher values of $I$ give higher line fluxes and in particular, more emission is seen in cores of the lines.

Figures 5a-f contain the flux profiles for the same parameters as those in Figs. $4 \mathrm{a}-\mathrm{f}$ but with $V_{B}=50 \mathrm{mtu}$. The P Cygni type profiles are obtained similar to those shown in Fig. 3.

Figures 6a-c give the variation of equivalent widths against the expansion velocity $V_{B}$ for the parameters shown in the figure. We can see that when there is no radiation incident from the companion, the equivalent widths are much larger as the absorption core is deeper. In the presence of dust $\tau_{\mathrm{d}}=1,2,5$ the equivalent widths reduce considerably for the reason given above that more photons are scattered into the core, thus reducing absorption and increasing core emission.
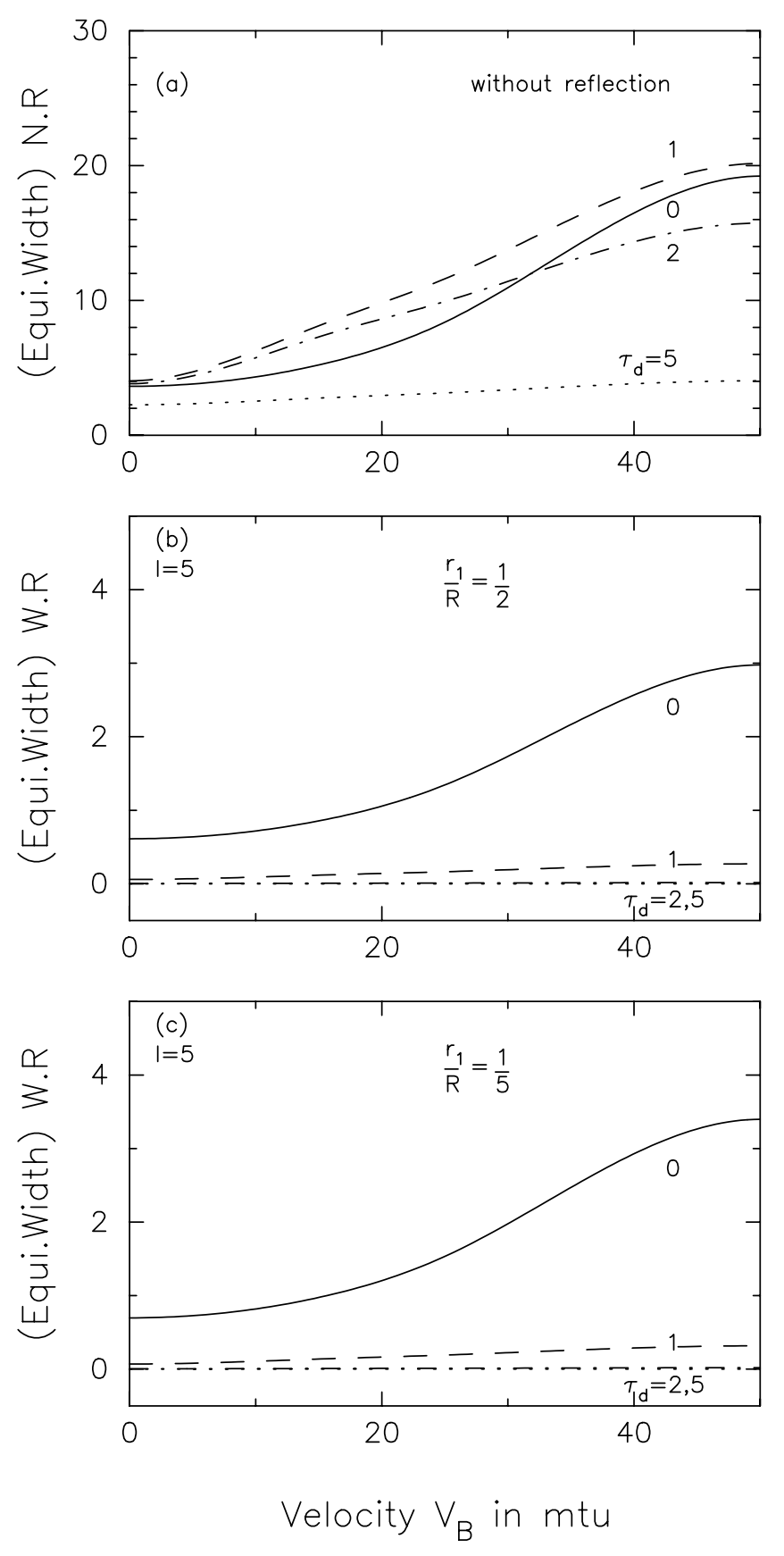

Fig. 6. Equivalent widths are plotted against the expansion velocity with reflection and without reflection

Figure 7 gives the variation of the ratio of height of emission $\left(H_{\mathrm{e}}\right)$ to that of depth absorption $\left(H_{\mathrm{a}}\right)$. There is no change between the reflection case and the nonreflection case. However it is noteworthy that this ratio reaches a maximum at about $V_{B}=4$ to $10 \mathrm{mtu}$ and then falls slowly as the expansion velocity increases. Similar kind of trend is observed in the presence of dust. It is different for $\tau_{\mathrm{d}}=5$ in that case the ratio increases slowly as the expansion velocity increases. This can be understood again from the argument that more dust scatters more 


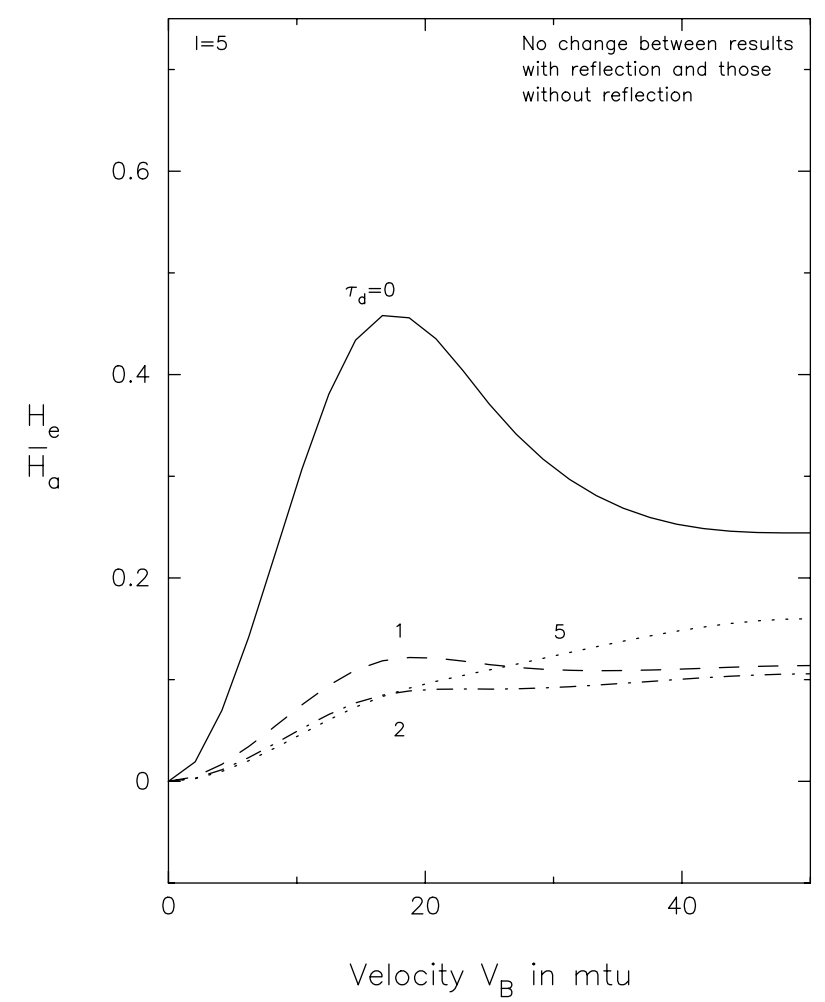

Fig. 7. The ratios of the heights of the emission to the depth of the absorption in the lines for both the case of reflected radiation and non reflected radiation are shown against the velocity expansion $V_{B}$

photons into the line core enhancing emission of the core of the line.

\section{Conclusions}

We have computed line profiles formed in a dusty and expanding spherical atmosphere of the components of a close binary system. We have considered dust scattering optical depth as high as 5 but no absorption due to dust is included. The scattering (isotropic) of dust increases the core emission whether the medium is static or moving. The condition of isotropic scattering should be replaced by Mie scattering phase function for correct estimation of dust scattering. Further, we need to study the formation of lines in the distorted shapes of the components which is under study.

\section{References}

Buerger P., 1969, ApJ 158, 1151

Buerger P., 1972, ApJ 177, 657

Claret, Gemenz, 1992, A\&A 256, 572

Nordlund A., Vaz L.P.R., 1990, A\&A 228, 231

Peraiah A., 1984, in Methods in Radiative Transfer, Kalkofen W. (ed.). Cambridge University Press, Cambridge

Peraiah A., Srinivasa Rao M., 1983, J. Astrophys. Astron. 4, 175

Peraiah A., Srinivasa Rao M., 1998, A\&AS 132, 45 (Paper I)

Peraiah A., Varghese B.A., Rao M.S., 1987, A\&AS 69, 345

Peraiah A., Wehrse R., 1978, A\&A 70, 213

Vaz L.P.R., 1985, Ap\&SS 113, 349

Wehrse R., Kalkofen W., 1985, A\&A 147, 71

Wilson R.E., 1990, ApJ 356, 613 